\title{
The Effect of Resource Aggregation at Different Scales: Optimal Foraging Behavior of Cotesia rubecula
}

\author{
Brigitte Tenhumberg, ${ }^{1,2, *}$ Michael A. Keller ${ }^{1, \uparrow}$ Andrew J. Tyre, ${ }^{1,2,}$ and Hugh P. Possingham ${ }^{2, \varsigma}$
}

1. Department of Applied and Molecular Ecology, University of Adelaide, Waite Campus, Private Bag 1, Glen Osmond, South Australia 5061, Australia;

2. Ecology Centre, Department of Zoology and Entomology, University of Queensland, St. Lucia, Queensland 4072, Australia

Submitted October 24, 2000; Accepted May 16, 2001

\begin{abstract}
Resources can be aggregated both within and between patches. In this article, we examine how aggregation at these different scales influences the behavior and performance of foragers. We developed an optimal foraging model of the foraging behavior of the parasitoid wasp Cotesia rubecula parasitizing the larvae of the cabbage butterfly Pieris rapae. The optimal behavior was found using stochastic dynamic programming. The most interesting and novel result is that the effect of resource aggregation within and between patches depends on the degree of aggregation both within and between patches as well as on the local host density in the occupied patch, but lifetime reproductive success depends only on aggregation within patches. Our findings have profound implications for the way in which we measure heterogeneity at different scales and model the response of organisms to spatial heterogeneity.
\end{abstract}

Keywords: resource aggregation, optimal foraging behavior, Cotesia rubecula, stochastic dynamic programming, spatial scale.

The importance of scale to ecological investigations has been demonstrated in many different areas, including behavioral ecology, population ecology, community ecology, and plant physiology (Wiens 1989). Ecologists are increasingly interested in ecological patterns and processes that are scale dependent, where patterns at one scale change when observed at other scales (Wiens 1989; Levin 1992; Schneider 1994). This article is concerned with a behav-

\footnotetext{
* Corresponding author; e-mail: btenhumberg@zen.uq.edu.au.

${ }^{\dagger}$ E-mail: mkeller@waite.adelaide.edu.au.

${ }^{\ddagger}$ E-mail: dtyre@zen.uq.edu.au.

`E-mail: hpossingham@zen.uq.edu.au.
}

Am. Nat. 2001. Vol. 158, pp. 505-518. (c) 2001 by The University of Chicago. 0003-0147/2001/15805-0005\$03.00. All rights reserved. ioral process. In particular, we examine the scale-dependent effects of resource aggregation on optimal patch-leaving behavior and reproductive success of consumers.

Previous work in optimal foraging theory concentrates on the influence of resource density within patches on patch residence time (e.g., Charnov 1976; Cook and Hubbard 1977; Comins and Hassel 1979; Iwasa et al. 1981; Visser et al. 1992; Nelson and Roitberg 1995). In general, these models are based on rate maximizing theory and predict that foragers leave a patch when its rate of fitness gain drops to a threshold value. However, the spatial arrangement of resource-containing patches is equally important for behavioral decisions (e.g., Lima and Zollner 1996; Roitberg and Mangel 1997).

The spatial arrangement of resources can vary at different scales. Resource-containing patches may be aggregated in space, while at a smaller scale, there may be aggregation in the frequency distribution of resource density within patches. Aggregation of resource-containing patches implies a spatial correlation between patches; there will be clusters of patches in space. Mangel and Adler (1994) proposed a description of spatial pattern based on Markov processes that describes aggregation at multiple spatial scales. Their "structure function" describes the probability that a point some distance $r$ away from a given point contains a resource, conditioned on the resource state at the current point. When resources are aggregated in space, the expectation of finding a resource close to an occupied patch is greater than the average probability of finding a resource over the entire habitat and greater than the expectation of finding a host close to an unoccupied patch. Structure functions can describe different degrees of spatial aggregation (Roitberg and Mangel 1997).

Aggregation of resource density within patches implies that the variance of the distribution is greater than the mean; there will be many patches with no resources and a few with a very high resource density. The frequency distribution of resource densities within patches (fig. 1) summarizes the resource availability in the environment. Resource availability influences the behavior of consumers, in particular, decisions concerning patch use and the dis- 


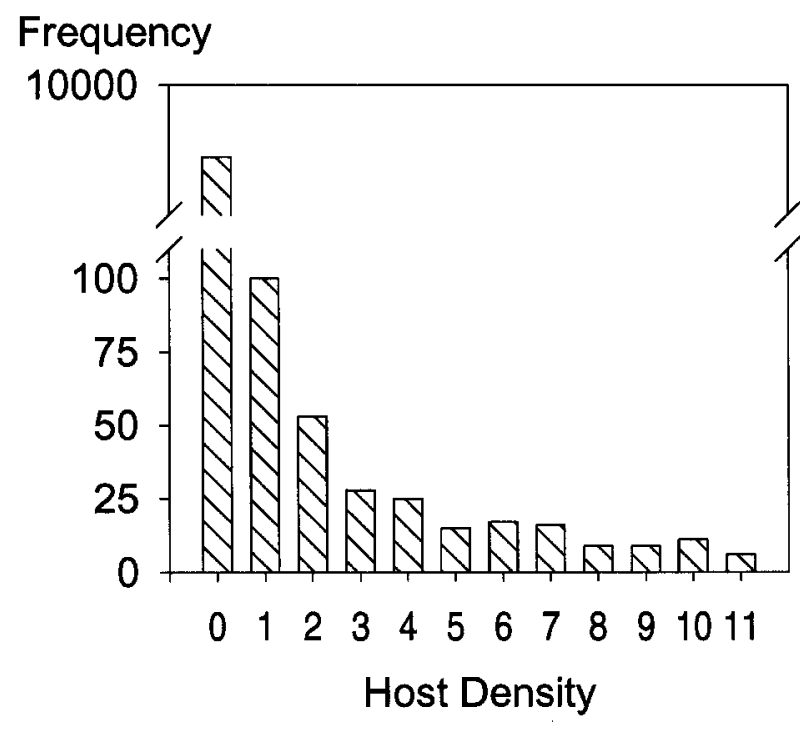

Figure 1: Frequency distribution of resource densities derived from a negative binomial distribution with mean $m=3$ and aggregation index $k=0.01$.

tribution of consumers between patches (Stephens and Krebs 1986; Bernstein et al. 1991). For decisions made at the individual level, however, the frequency distribution of resources within patches alone might be insufficient because those decisions might also be influenced by the arrangement of resource-containing patches in space. For example, the resources described by the frequency distribution of figure 1 can be arranged randomly or aggregated in space (fig. 2). Imagine a forager in a habitat where resource patches are aggregated in space (fig. $2 A$ ). In this case, the probability of encountering a resource patch nearby will be higher than average if the forager is already in a resource patch. The encounter probability is lower if the forager is in an empty patch. Therefore, the distribution of resources in space influences the travel time. In the presence of resource depletion, we expect a forager to spend less time in a patch when the average travel time is short because short travel times translate to a high average rate of encountering resource patches (the marginal value theorem of patch use; Charnov 1976). The spatial distribution of resources influences the variation of travel times experienced by the foragers (e.g., travel time as a function of resource status of the current patch). Variation in encounter rates with resources can have consequences for life-history decisions (Tenhumberg et al. 2000). In this article, we examine whether variation in travel times influences the patch-leaving behavior as well.

We study how the two different scales of aggregation influence the patch-leaving behavior of foragers. For the remainder of the article, we refer to density aggregation when describing the aggregation of resources within patches and to spatial aggregation when describing the aggregation of resource-containing patches in space. Increasing density aggregation arises from increased variance of the frequency distribution of resources within patches. Increasing spatial aggregation arises from increasing the spatial correlation between patches that contain resources.

Consider a three species parasitoid-herbivore-plant system in which plants (i.e., resource patches) are distributed throughout the landscape and in which zero, one, or more herbivores are present on each plant. How does the spatial clustering of resource-containing plants (i.e., plants with herbivores) and the distribution of herbivores among plants influence the parasitoid behavior and reproductive success? Our article addresses this question for the parasitoid wasp Cotesia rubecula. We develop a stochasticstate-dependent optimal foraging model of foraging behavior of the parasitoid wasp C. rubecula parasitizing the larvae of the cabbage white butterfly Pieris rapae. An unexpected and novel result is that the effect of aggregation on patch residence time and lifetime reproduction depends on whether hosts are aggregated within or between patches. High spatial aggregation of host-containing patches causes shorter patch residence times at all host densities, whereas density aggregation decreases patch residence time on plants when host density is low and increases patch residence time on plants when host density is high. Lifetime reproduction is more sensitive to density aggregation than spatial aggregation. Whereas the wasp's patch-leaving behavior compensates completely the effect of spatial aggregation on lifetime reproduction, increased density aggregation still reduces lifetime reproduction.

\section{The Biological System}

Cotesia rubecula females are solitary parasitoid wasps that lay single eggs inside their hosts. After hatching, a wasp larva feeds internally on the host's tissue and kills the host at the end of larval development. The hosts are larvae of the cabbage white butterfly, which feed and live on cruciferous plants. Plants change the amount and composition of odor constituents in response to herbivore damage. These infochemicals are attractive to the wasps (Nealis 1986). It has been demonstrated for some parasitoid species that females can distinguish patches with different host densities based on the concentration of infochemicals (van Alphen and Vet 1986). When a wasp arrives on a plant, she starts searching for hosts in the vicinity of areas with host-feeding damage (Nealis 1986). However, feeding damage does not always indicate the presence of hosts. Hosts move away from feeding sites while resting or molting to avoid predators. 

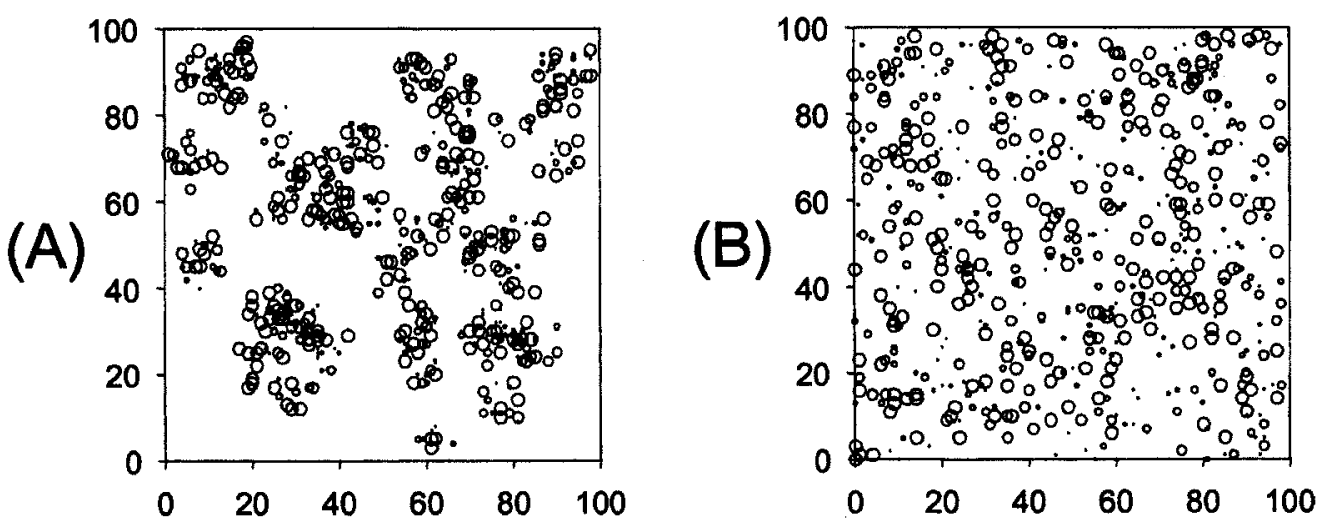

Figure 2: Two distributions of resources in space: $(A)$ aggregated and $(B)$ random. Each point in the grid is a patch; there are 10,000 patches in all. Patches with resource densities $>0$ are displayed by a circle. The diameter of the circle is proportional to the density in that sampling unit. In both cases, the frequency distributions of resource densities can be described by the same negative binomial distribution (fig. 1).

Wasps readily attack hosts they encounter (M. A. Keller, personal observation), even if the hosts are already parasitized (superparasitism). As C. rubecula is a solitary parasitoid, only one egg can develop within a single host (Godfray 1987). Therefore, the profitability of a plant decreases with time because the probability of encountering and ovipositing in an unparasitized host decreases with each oviposition. Eventually the wasp leaves the plant to search for hosts elsewhere.

The patch-leaving behavior of parasitoids has attracted a lot of attention by researchers (e.g., Hubbard and Cook 1978; Waage 1979; Iwasa et al. 1981; Haccou and Hemerik 1985; Haccou et al. 1991; Roitberg et al. 1992; Hemerik et al. 1993; van Alphen 1993; Wiskerke and Vet 1994; Driessen et al. 1995). The result of this extensive research indicates that the probability of a wasp leaving a patch (e.g., plant) generally depends on several factors, including the parasitoid's age, the patch residence time, the host density in the current patch, the density and distribution of hosts in the rest of the habitat, and the number of ovipositions or the oviposition rate in the current patch. A single-scale perspective characterizes all this research. Our model incorporates all the aforementioned factors, but in addition, it includes the influence of host distribution on multiple scales.

\section{The Model}

We used stochastic dynamic programming (SDP; Bellman 1957; Mangel and Clark 1988) to predict when a parasitoid should leave a patch. In SDP models, behaviors maximizing the fitness of organisms are identified as a function of an organism's internal and external state(s), the remaining life expectancy, and physiological and ecological parameters. SDP models start at the end of an individual's life and then work backward in time, calculating for each combination of states the behavior that results in the highest fitness. After calculating the optimal behaviors, we use a Monte Carlo simulation to analyze the foraging behavior of female wasps using decisions determined by the SDP.

For the sake of simplicity, we consider only mated female wasps. The wasps are foraging in a grid of uniformly distributed patches. The patches are either empty or contain up to 10 hosts. The location of empty patches determines the degree of spatial aggregation, while the distribution of hosts within nonempty patches determines the degree of density aggregation.

The model consists of two parts: searching for hosts on a patch (i.e., cabbage plant) and flying between patches searching for suitable landing sites. On the patch, there is the following hierarchy of events. If a wasp survives the next time step of length 2.5 min (survival probability $=1-$ $\mu_{1}$ ), she has to decide whether to stay or to leave the patch. If she stays, there is the probability $\psi$ that she encounters a host. She attacks all hosts she encounters, but successfully oviposits an egg only with probability $s$. She must handle the host for $h$ time steps regardless of whether oviposition is successful. Every time step a wasp survives outside a patch (survival probability $=1-\mu_{2}$ ), she encounters a new patch on which she may or may not land. The number of patches encountered since last leaving a patch is the travel distance. The probability of landing $\beta_{d}$ depends on the host density $d$ in that particular patch (M. A. Keller, unpublished data).

\section{Model Assumptions}

Fitness Currency. We assume that Cotesia rubecula females adjust their foraging behavior to maximize their fitness 
(Mangel and Clark 1988). In our model, we use the total number of surviving eggs oviposited by a female during her life as fitness currency. Other models used the expected initial egg complement of daughters (the "grand eggs") as a fitness measurement (Heimpel et al. 1998), taking into account that larger hosts generally produce parasitoids with higher egg load. This fitness currency is appropriate for parasitoids that reject some hosts for oviposition. In contrast, C. rubecula females try to oviposit in all hosts they encounter (M. A. Keller, personal observation). As our model is concerned only about patch-leaving decisions, we ignore the influence of host size on a parasitoid's fitness, even though the instar of the host also influences the fitness of the parasitoid's offspring (Nealis et al. 1984).

State Space. We assume that a parasitoid's patch-leaving decision depends on the following state variables: her age, the host density and distribution in the habitat, the host density in the current patch, how long she has searched (patch residence time), and how many eggs she has laid in the current patch. We assume that wasps have perfect knowledge of all these states. The patch residence time and the number of ovipositions in that patch are the minimally sufficient statistics a forager needs in order to estimate the number of unparasitized hosts remaining in the patch (Iwasa et al. 1981). As C. rubecula is probably not egg limited (Nealis 1990), we assume ovipositions in previous patches have no influence on her patch-leaving behavior.

Patch Depletion. We include only depletion as a result of a wasp's foraging behavior, such that the probability of encountering an unparasitized host in a patch is a decreasing function of the number of ovipositions. We assume a system with a high number of patches and a low number of parasitoids, such that wasps never encounter an already depleted patch. The probability of reencountering a previously visited patch or a patch depleted by a conspecific is negligible. The effect of allowing other wasps

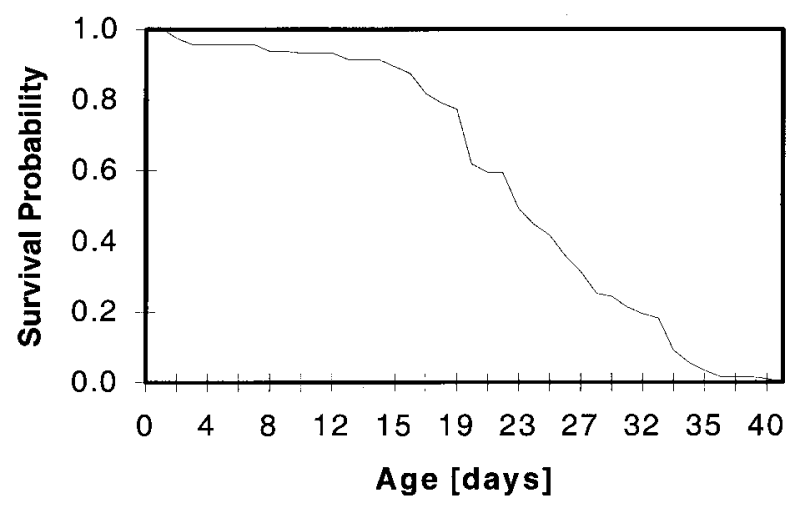

Figure 3: Daily probability of survival as a function of age $\left(\mu_{1}(t)\right)$ for female Cotesia rubecula, estimated from laboratory data (Nealis et al. 1984).

to oviposit in the patch would be to lower the expected payoff from each host by a uniform amount if females cannot detect each other's ovipositions.

Survival. In our model, we derive the mortality risk of wasps in a patch from the survival curves measured under laboratory conditions by Wäckers and Swaans (1993), with an average life expectancy of $23 \mathrm{~d}$. The cost of searching for suitable landing sites involves time loss and an increased mortality risk. Roff (1977) showed that dipterans incur a higher mortality rate flying rather than walking, which in turn reduces the average lifetime expectancy. To account for the increased mortality risk during flight, we multiplied the mortality risk in a patch by a fixed factor (table 1; fig. 3).

The life expectancy of wasps in the real world is probably much shorter than that of our model wasps. Potential mortality sources for adult parasitoids in the field include abiotic factors (DeBach et al. 1955; Roitberg et al. 1992; Fink and Völkl 1995), starvation (Wäckers and Swaans

Table 1: Parameter values and functions included in the stochastic dynamic programming model

\begin{tabular}{|c|c|c|c|}
\hline & Parameter & Value or formula & Reference \\
\hline$\beta_{d}$ & Prob $\{$ landing $\mid \mathrm{d}\}$ & $\frac{\exp -1.0613+0.1125 d}{1+\exp -1.0613+0.1125 d}$ & M. Keller, unpublished data \\
\hline$\mu_{1}(t)$ & Mortality rate in patch & 1 - survival probability (fig. 3 ) & Wäckers and Swaans 1993 \\
\hline$\mu_{2}(t)$ & Mortality rate while flying & $1-($ survival probability $\times .99)$ & \\
\hline$\psi_{\text {good }}(d)^{\mathrm{a}}$ & $\begin{array}{l}\text { Prob }\{\text { encounter an unparasitized } \\
\text { host } \mid d\}\end{array}$ & $\begin{array}{l}\mathrm{f}(\text { number of ovipositions, } d) \\
\quad(\text { table } 3)\end{array}$ & \\
\hline$\psi_{\mathrm{bad}}(d)^{\mathrm{a}}$ & $\begin{array}{l}\text { Prob }\{\text { encounter a parasitised } \\
\text { host } \mid d\}\end{array}$ & $1-\exp (-d / 7)-p_{\text {good }}$ & \\
\hline$\psi(d)^{\mathrm{a}}$ & $\operatorname{Prob}\{$ encounter a host $\mid d\}$ & $\psi_{\text {good }}(d)+\psi_{\text {bad }}(d)$ & \\
\hline$s$ & $\operatorname{Prob}\{$ oviposition $\mid s\}$ & .84 & Nealis 1986 \\
\hline
\end{tabular}

Note: $d=$ host density in current patch, $t=$ age of the parasitoid.

${ }^{a}$ Assumes that host encounters in patch follow a Poisson distribution. See text for formula and derivation of $\psi_{\text {good }}(d)$. 
1993; Heimpel and Collier 1996; Jervis et al. 1996), and predation (Rees and Onsager 1982; Völkl 1992; Völkl and Mackauer 1993; Rosenheim et al. 1995). For example, the longevity of Trichogramma platneri in the laboratory is reduced $90 \%$ by unfavorable temperature and $75 \%$ by starvation (McDougall and Mills 1997). Similar drastic effects of starvation on the life expectancy of C. rubecula have been reported (Wäckers and Swaans 1993; G. Siekmann, unpublished data). Actual field mortality rates from any of these factors are largely unknown (Heimpel et al. 1997). However, field experiments on Aphytis parasitoids suggest that predation rivals starvation and extreme temperature conditions as sources of mortality (Heimpel et al. 1997). We do not expect that the qualitative results of our model will be affected by choosing a life expectancy that is too long. Simulations with a drastically reduced life expectancy $(2 \mathrm{~d})$ produced a similar distribution of patch times; the lifetime offspring production was scaled down without changing the relative differences in reproduction resulting from differing degrees of aggregation.

Activity Period. In our model, the wasps are active $12 \mathrm{~h}$ each day. This activity period is probably too high; real wasps spend at least $50 \%$ of their time resting and grooming and are inactive during unsuitable weather (Nealis 1986; Geervliet 1997; M. A. Keller, personal observation). Unfortunately, no data are available on the proportion of time wasps spend foraging in the field. A shorter activity period would reduce the life expectancy and consequently scale the reproductive success down further.

No Egg Limitation. In our model, female C. rubecula are not egg limited and try to oviposit in all hosts encountered. A check on this assumption is the magnitude of the lifetime egg production of simulated wasps. At a mean host density of two hosts per plant, model wasps laid fewer than 400 eggs during their life. As Nealis (1990) found an average of 100 mature eggs in the uterus of C. rubecula females, this egg production is above the natural egg complement of the wasps. However, our model wasps probably have at least a tenfold longer life expectancy than real wasps (average life expectancy of model wasps $=23 \mathrm{~d}$, compared with $1-4 \mathrm{~d}$ at $>20^{\circ} \mathrm{C}$ in field cages; G. Siekmann, unpublished observations). Therefore, we think that real wasps hardly ever run out of eggs.

Superparasitism. Our model assumes that female wasps cannot distinguish parasitized from unparasitized hosts, and as a consequence, wasps attack every host they encounter. Acceptance of parasitized hosts could result from one of two mechanisms: adaptive superparasitism (van Alphen et al. 1992; Visser et al. 1992; Papaj and Messing 1996; van Randen and Roitberg 1996) or an inability to distinguish between parasitized and unparasitized hosts (Rosenheim and Mangel 1994).

In our model, all eggs oviposited in previously parasitized hosts die. We assume that only a single wasp forages in each patch, so it does not matter which egg survives when superparasitism occurs; all that matters is that the potential payoff from a patch decreases with time as the number of unparasitized hosts decreases. If adaptive superparasitism operates in C. rubecula, then ovipositing more than one egg in a single host would increase the fitness payoff. However, deliberate self-superparasitism in solitary insects is rather unlikely (Godfray 1994).

Host Distribution. The model includes three different host distributions: the distribution of hosts within a patch, the distribution of host densities among patches, and the spatial distribution of patches. We assume hosts are randomly distributed within a patch. Therefore, we model the probability that wasps encounter a host in any particular time step as a Poisson process. We assume that the frequency distribution of host densities within plants (e.g., the distribution of circle sizes in fig. 2) comes from a negative binomial distribution. We assume the spatial distribution of host-containing patches (e.g., spatial arrangement of all circles in fig. 2) comes from a one-dimensional structure function.

Using a one-dimensional structure function to describe the spatial distribution of host-occupied patches assumes that the host densities of neighboring patches are not correlated. In many species, host densities on neighboring plants are highly correlated. Such a high correlation occurs particularly when reproduction and juvenile development take place in adjacent places, such as in aphid populations. In this case, the description of aggregation in space is more complicated than we have discussed here (e.g., Possingham et al. 1994). However, there are populations, such as for larval Pieris rapae, where the assumption of uncorrelated host densities in neighboring patches is realistic. Pieris rapae females are very mobile and fly randomly among brassicaceous plants where they lay mostly one or two, but sometimes up to five, eggs per plant (Harcourt 1961; Kobayashi 1966; Jones 1977; Kareiva and Shigesada 1983). The randomly distributed plant visits compounded with the logarithmically distributed number of eggs laid at each visit generates a negative binomial distribution of eggs per plant in cabbage fields (Kobayashi 1966). The feeding activity of predators, or heterogeneous light conditions (Kobayashi 1966), might result in a spatially aggregated distribution of host-infested plants. Unfortunately, no data on the spatial distribution of larval P. rapae in their natural habitat have been published. 


\section{Characterizing Density and Spatial Host Distributions}

The probability of encountering a host-occupied patch depends on the distance $r$ the wasp has covered since she left the patch. Every time step a wasp is outside a patch, she encounters a new patch with $d$ hosts, and she lands with the probability $\beta_{d} ; r$ is then the total number of patches encountered since last leaving a patch. The probability that this patch is host occupied (i.e., $d>0$ ) is given by the structure function, that is, the conditional probability that a patch is host occupied given the last patch at distance $r$ was host occupied $(p[r \mid 1])$ or was not host occupied $(p[r \mid 0])$. Figure 4 (modified fig. 1 from Mangel and Adler 1994) illustrates hypothetical structure functions for random and aggregated patterns similar to those in figure 2.

We derived $p(r \mid 1)$ from a one-dimensional first-order Markov process with three parameters, $p_{\mathrm{a}}$, the average patch density, $p_{0}$ and $p_{1}$ (Mangel and Adler 1994):

$$
\begin{aligned}
& p(r \mid 1)=p_{\mathrm{a}}+\left(1-p_{\mathrm{a}}\right)\left(p_{1}-p_{0}\right)^{r}, \\
& p(r \mid 0)=\frac{p_{\mathrm{a}}[1-p(r \mid 1)]}{\left(1-p_{\mathrm{a}}\right)}
\end{aligned}
$$

where $p_{1}$ is the probability that a patch immediately next to the current host-occupied patch $(r=1)$ is also occupied and $p_{0}$ is the probability that the next patch is unoccupied. The parameters $p_{1}$ and $p_{0}$ are related by the equation

$$
p_{\mathrm{a}} p_{1}+\left(1-p_{\mathrm{a}}\right) p_{0}=p_{\mathrm{a}}
$$

Equation (1) implies that $p_{1}\left(0<p_{1}<1\right)$ and the degree of spatial aggregation are positively correlated. Hence, we can examine the effect of spatial aggregation on parasitoid behavior simply by varying $p_{1}$. For further details on the structure function, see Mangel and Adler (1994).

The spatial and density distributions are connected by $p_{a}$, which is set to 1 minus the zero term of the negative binomial distribution. In our model, a patch can contain zero to 10 hosts. In the field, usually a cabbage plant contains between zero and two P. rapae larvae (Harcourt 1961; Kobayashi 1966; Jones 1977). The negative binomial distribution has two parameters: the mean host density, $m$, and the aggregation index, $k$. In general, the distribution becomes more aggregated as $k$ becomes smaller. The probability that a host-occupied patch contains $d$ hosts is

$$
H_{d}=\left[\frac{\Gamma(k+d)}{d ! \Gamma(k)}\right]\left(\frac{m}{m+k}\right)^{d}\left(\frac{k}{m+k}\right)^{k},
$$

where $\Gamma(k)$ is the gamma function (Krebs 1989).

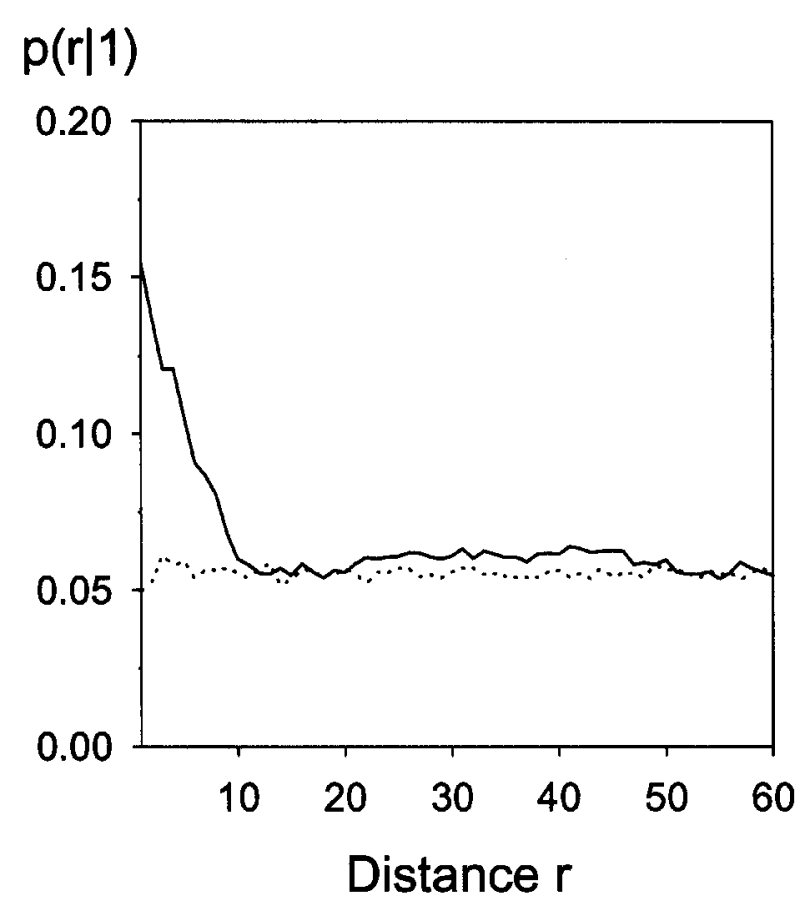

Figure 4: Probability of encountering a resource as a function of distance $r$ from any given point in the habitat given the point contains a resource $(p[r \mid 1])$. The average probability of finding a patch with resource $\left(P_{\mathrm{a}}\right)$ is 0.055 ( $1-P_{0}$; same parameters as fig. 2). Broken line, aggregated pattern (fig. 2A); solid line, random pattern (fig. $2 B$ ).

If $p_{1}$ is very small $\left(p_{1}=0.1\right)$, the distribution of hostoccupied patches is completely random, and the spatial and density distributions could be described by the negative binomial distribution alone. In this respect, we can consider the negative binomial distribution a special case of the "combined" distribution used in our model.

The density distribution of $P$. rapae eggs among plants is usually aggregated with $k$ values $<1$ (Jones 1987). In this article, we examine $k$ values between 1 and 0.01 , which are consistent with $k$ values found in natural insect populations (table 2).

The probability of finding $d$ hosts $r$ patches away is

$$
\begin{aligned}
\lambda_{r, 0} & =p(r \mid 0), \\
\lambda_{r, d} & =p(r \mid 1) H_{d}, \\
d & =1, \ldots, 10 .
\end{aligned}
$$

When both spatial and density aggregation are low, that is,

$$
p_{1} \leq p_{\mathrm{a}}=[1-n(0)]
$$

where $n(0)$ is the number of empty patches, then the struc- 
Table 2: Density aggregation in empirical studies

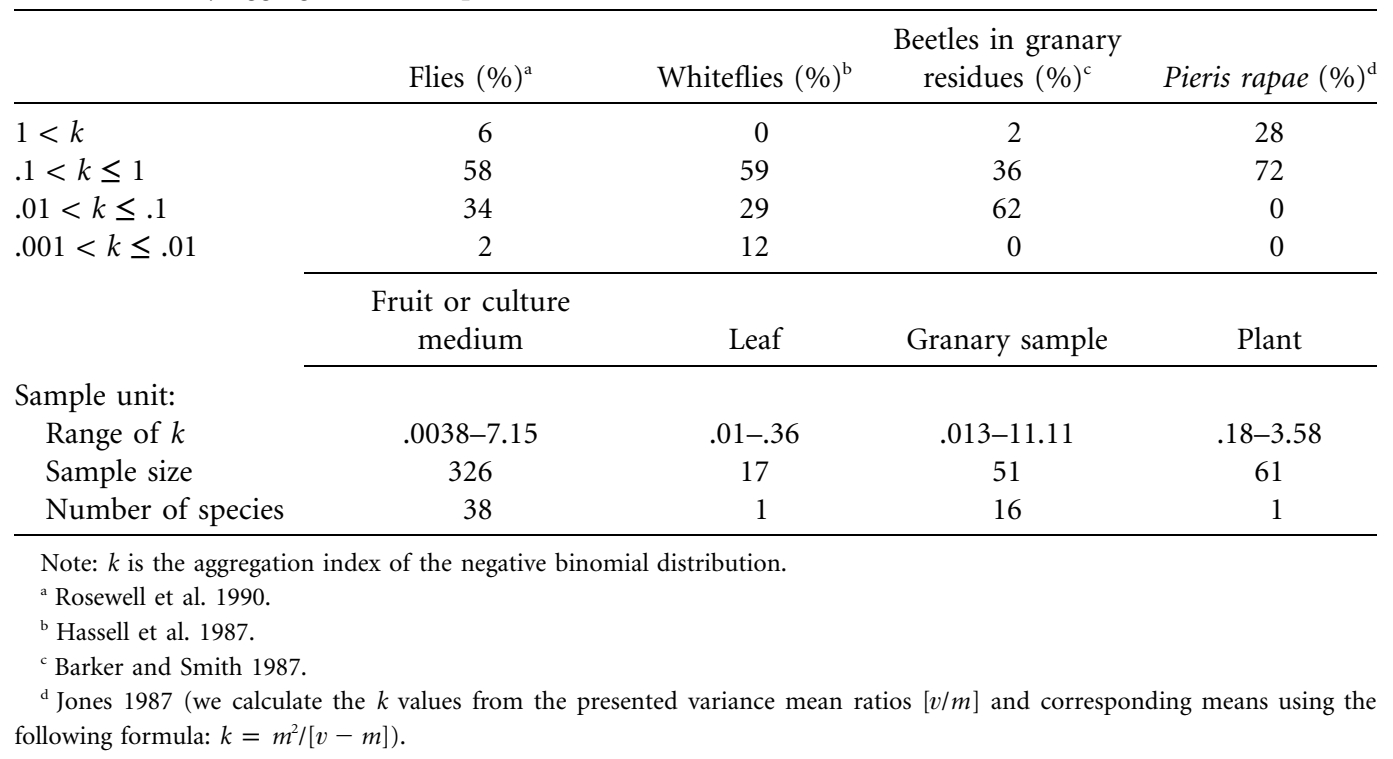

ture function we have used here yields overdispersed patterns. Combinations of parameters that meet this condition have been excluded from the results (i.e., $p_{1}<0.5$ when $k=1)$.

The type of spatial structure described by our combination of a structure function and a negative binomial distribution is similar to a "marked-point process" (Stoyan and Stoyan 1994), where a random process creates the points (i.e., occupied patches) and a second random process creates marks (i.e., the number of hosts) attached to each point. The statistical methods for dealing with marked-point processes are good at providing top-down descriptions of pattern, but we require a bottom-up wasp's eye view of the spatial structure for our model, and structure functions effectively provide this.

\section{Dynamic Programming Equation}

Given one time step is $2.5 \mathrm{~min}$ (both inside and outside patches) and assuming that a wasp forages $12 \mathrm{~h} / \mathrm{d}$ during her maximum life span of $40 \mathrm{~d}$ (Wäckers and Swaans 1993), then the maximum foraging time of a wasp is 11,520 time steps. The SDP model includes the state variable time $(t=1,2, \ldots, 11,520)$ and the patch-related variables host density $(d=0,1,2, \ldots, 10)$, patch time $\left(t_{\mathrm{p}}=1,2, \ldots, 17\right)$, and number of ovipositions ( $e=$ $0,1,2, \ldots, 17)$; therefore, the lifetime fitness function is defined as $F\left(t, d, t_{\mathrm{p}}, e\right)$. A wasp in a patch may leave or stay in the patch; each decision translates in a particular fitness payoff ( payoff $_{\text {leave }}$, payoff stay $_{\text {) }}$ ).

After leaving a patch, the wasp encounters another patch every time step on which she may or may not land. The probability of landing on a particular patch, $\beta_{d}$, depends on the host density $d$ of the patch. The parameter $\beta_{d}$ is estimated from a logistic regression on unpublished data from M. A. Keller. He placed 16 cabbage plants containing different host densities $(0,1,2$, and 5 hosts/plant) in the field and observed the landing behavior of C. rubecula foraging between those plants. The probability of finding a plant with a certain host density $d$ after covering the distance $r, \lambda_{d, r}$ is determined by the distribution of host densities between patches and the spatial distribution of host-occupied patches (see eq. [4]).

While in a patch, a wasp encounters a host with the probability of $\psi(d)$ in which she successfully oviposits an egg with the probability s. Independent of the oviposition success, she spends an extra time step to handle the host. The payoffs for encountering an unparasitized ( host $_{\text {good }}$ ), parasitized (host $\mathrm{bad}_{\text {) }}$ ), or no host $\left(\right.$ host $\left._{0}\right)$ are as follows:

$$
\begin{aligned}
\operatorname{host}_{\text {good }}= & s\left[F\left(t+2, d, t_{\mathrm{p}}+2, e+1\right)+1\right] \\
& +(1-s) F\left(t+2, d, t_{\mathrm{p}}+2, e\right), \\
\text { host }_{\mathrm{bad}}= & s F\left(t+2, d, t_{\mathrm{p}}+2, e+1\right) \\
& +(1-s) F\left(t+2, d, t_{\mathrm{p}}+2, e\right), \\
\text { host }_{0}= & F\left(t+1, d, t_{\mathrm{p}}+1, e\right) .
\end{aligned}
$$

Note that successful oviposition in an already-parasitized host changes the state space but provides no fitness increment.

The scenario described above is described with the following dynamic programming equation where fitness is optimized over the biological choice, leave or stay: 


$$
\begin{aligned}
& F\left(t, d, t_{\mathrm{p}}, e\right)= \max \left(\text { payoff }_{\text {leave }}, \text { payoff }_{\text {stay }}\right), \\
& \text { payoff }_{\text {leave }}=\sum_{r=1}^{10}\left\{\left[1-\mu_{2}(t+r)\right]\right. \\
& \quad \times \prod_{i=0}^{r-1}\left(1-\sum_{j=1}^{10} \beta_{j} \lambda_{j, i}\right) \\
&\left.\quad \times \sum_{d=1}^{10} F(t+r, d, 1,0) \lambda_{r, d} \beta_{d}\right\} \\
& \text { payoff }_{\text {stay }}=\left[1-\mu_{1}(t)\right]^{2} \\
& \times\left[\text { host }_{\text {good }} \psi_{\text {good }}(d)\right. \\
&\left.+ \text { host }_{\text {bad }} \psi_{\text {bad }}(d)\right] \\
&+\left[1-\mu_{1}(t)\right] \\
& \times \text { host }_{0}\left[1-\psi_{\text {good }}(d)-\psi_{\text {bad }}(d)\right],
\end{aligned}
$$

where $\mu_{1}(t)$ and $\mu_{2}(t)$ are the mortality rates in the patch and while flying, with $\mu_{1}(t)<\mu_{2}(t)$ and $\psi_{\text {good }}(d)$ or $\psi_{\text {bad }}(d)$ the probabilities of encountering an unparasitized or parasitized host. The values of these parameters and the references from which the values are derived are given in table 1.

The probability of encountering an unparasitized host, $\psi_{\text {good }}$, depends on the number of eggs that have already been laid and the number of hosts into which they have been laid, assuming that eggs are randomly distributed among the available hosts (table 3 ). The key is the probability distribution of the number of parasitized hosts on the plant $A$. This can be calculated iteratively for each host density using the observation that a particular number of parasitized hosts can arise only in two ways if hosts are parasitized one at a time. First, if the encountered host is unparasitized, then there will be one more parasitized host. Second, if the encountered host is parasitized, then the number of parasitized hosts will not change. The probability of $i$ parasitized hosts after $e$ eggs have been laid is

$$
A_{i, e}^{\prime}=A_{i, e-1} \frac{i}{d}+A_{i-1, e-1} \frac{d-i+1}{d} \text {, }
$$

where $d$ is the number of hosts in the patch. The initial distribution for $e=0$ is

$$
A_{i, 0}=\left\{\begin{array}{ll}
1, & \text { if } \quad i=0 \\
0, & \text { if } \quad i \geq 1
\end{array} .\right.
$$

We use this to calculate $\psi_{\text {good }}$ for a patch with $d$ hosts and $e$ eggs already laid as the sum of the probability of encountering an unparasitized host when $i$ hosts have been parasitized multiplied by the probability that $i$ hosts have been parasitized:

$$
\begin{aligned}
& \psi_{\text {good, } d, e}=\sum_{i=1}^{d} \frac{d-i}{d} A_{i, e}^{\prime} \\
& \psi_{\text {good }, d, 0}=1 \forall d \geq 1
\end{aligned}
$$

By applying equations (14) and (15) iteratively from $e=1$ up to the maximum number of eggs laid in a patch, we get all the probabilities required. (See Rosenheim and Mangel 1994 for another method of representing host depletion without recognition.)

\section{Results}

We analyze the effect of host aggregation at two scales on a parasitoid's patch residence time and lifetime reproduction: density aggregation (variance of the frequency distribution of host densities) and spatial aggregation. We ran the model for different mean host densities in the range of 0.1 and nine hosts per patch. The higher the mean density $m$ used, the higher the lifetime reproduction of the wasps became and the more readily the wasps left the patch. The influence of density aggregation and spatial aggregation on the patch-leaving behavior was qualitatively the same over the whole range of $m$. Therefore, we present the results only for a mean density of two hosts per patch, which is commonly observed in field populations. Figure

Table 3: Probability of encountering an unparasitized host as a function of host density $(d)$ in the patch (number of hosts) and the number of eggs already laid

\begin{tabular}{lcccc}
\hline Number of hosts & No eggs laid & One egg laid & Two eggs laid & $\ldots$ \\
\hline 0 & 0 & 0 & 0 & $\ldots$ \\
1 & 1 & 0 & 0 & $\ldots$ \\
2 & 1 & .5 & .25 & $\ldots$ \\
$\vdots$ & $\vdots$ & $\vdots$ & $\vdots$ & \\
$d$ & 1 & $\frac{d-1}{d}$ & $\sum_{i=1}^{d} \frac{d-i}{d} A_{i, e}^{\prime}$ & $\ldots$ \\
\hline
\end{tabular}



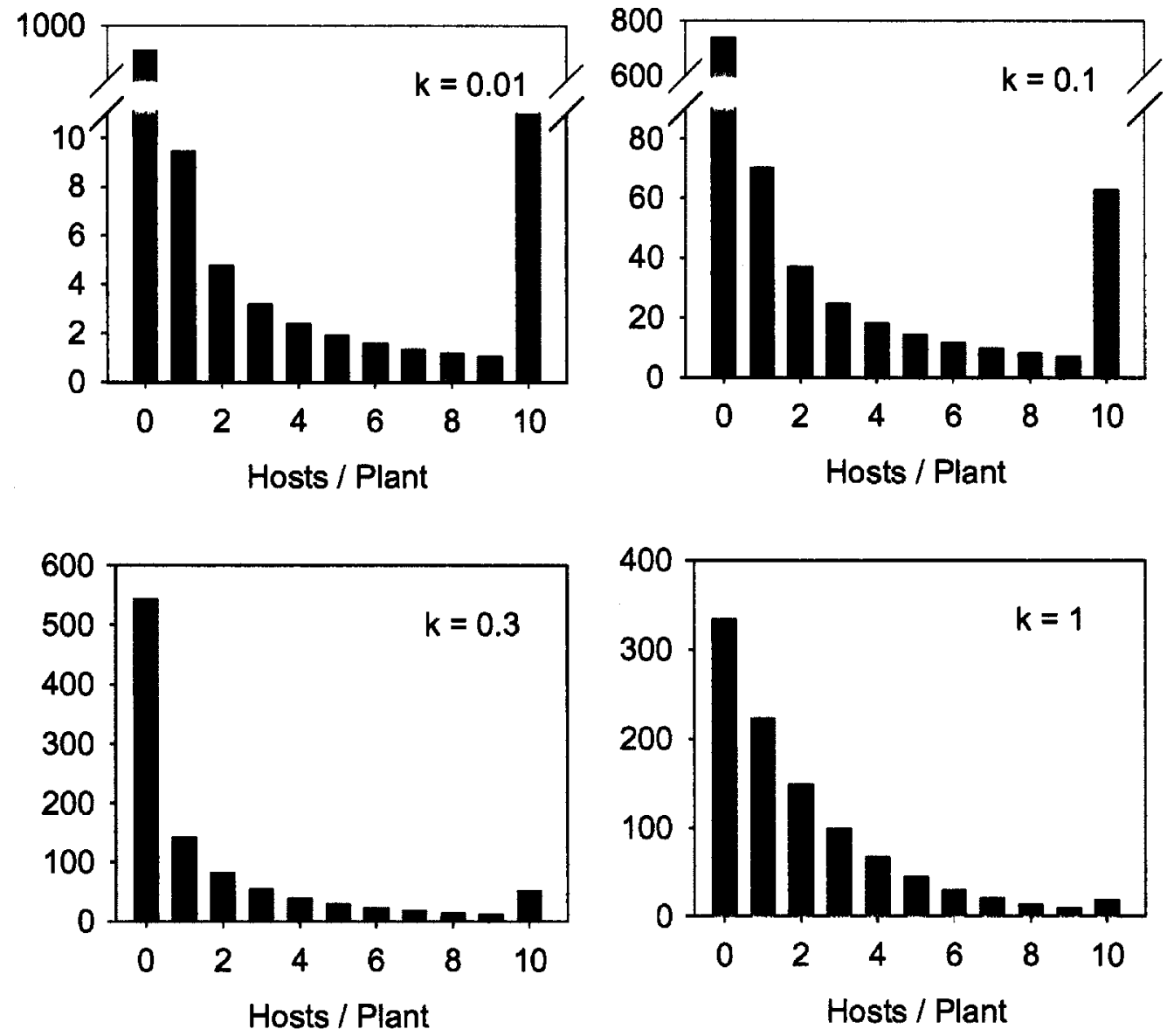

Figure 5: Frequency distribution of host densities within 1,000 host-occupied patches if the host densities have a negative binomial distribution with a mean host density $m=2$ and varying aggregation index $k$.

5 shows example frequency distributions of hosts within occupied patches for different degrees of density aggregation ( $k$ values).

\section{Patch Residence Time}

The response to aggregation is complex. In general, with increasing degree of aggregation, the average patch residence time decreases, independent of the scale of aggregation (fig. 6). However, at $k$ values $>0.1$, the effect of density aggregation is very small compared with spatial aggregation. The average time wasps spent on a patch during their life is the result of the wasps' patch-leaving behavior and the encounter frequency of patches with different host densities. Therefore, the average patch residence time does not necessarily reflect the behavior of wasps on patches with different host densities.

The influence of aggregation on the patch residence time depends on the host density of the current patch. Increas- ing host density reduces the magnitude of the effect of spatial aggregation but actually changes the direction of the effect of density aggregation (fig. 7). When a wasp is on a patch with low host density, patch residence time is shorter at higher degrees of spatial and density aggregation (fig. 7A). However at the extreme ends of spatial aggregation, that is, $p_{1}=0.1$ or 0.9 , there is no effect of density aggregation. On patches with higher host densities, the effect of aggregation at both scales disappears in the range of density aggregation found in natural Pieris rapae populations $(k>0.1$; fig. $7 B)$. However, at very high degrees of density aggregation $(k<0.1)$, wasps remain longer in a patch rather than leaving earlier as they do in patches with low host density. This interesting behavioral switch occurs within a range of density aggregation typical for a lot of insect species (table 2). Increasing spatial aggregation still causes wasps to leave earlier, but the magnitude of the effect is small. 


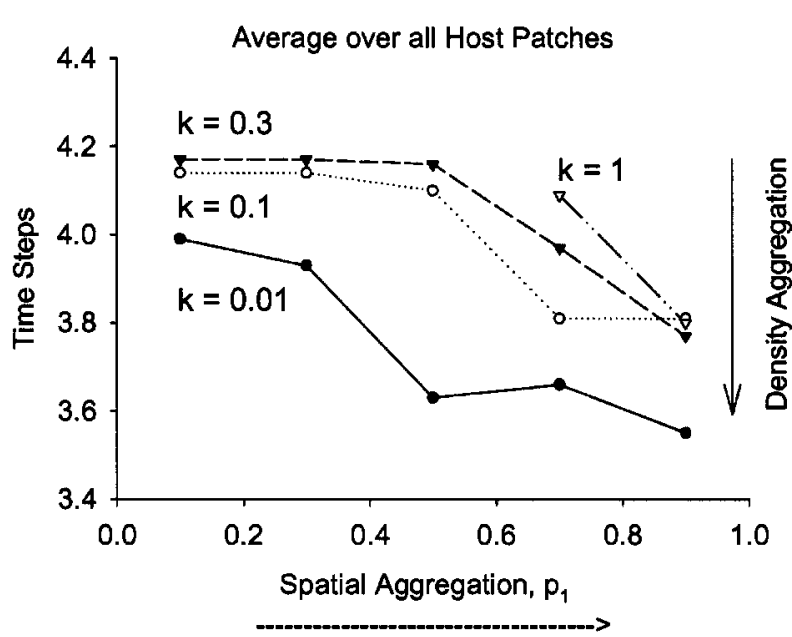

Figure 6: Influence of density aggregation $(k)$ and spatial aggregation $\left(p_{1}\right)$ on mean patch residence time of simulated Cotesia rubecula females averaged over all patches. The arrows indicate increasing degree of aggregation.

\section{Lifetime Reproduction}

As a result of adjusting their patch-leaving behavior in response to the spatial distribution of host-infested plants, the reproductive success of female Cotesia rubecula is independent of spatial aggregation (fig. 8). In contrast, with increasing degree of density aggregation, lifetime offspring production decreases. A high degree of density aggregation translates into a drastic increase in the frequency of empty patches (fig. 5). Therefore, with increasing degree of density aggregation, they waste an increasing amount of time visiting and resting on empty patches. Even though wasps always leave empty patches after one time step, patches with hosts are rare at higher degrees of density aggregation. Consequently, there are fewer opportunities to compensate for increased travel time.

\section{Discussion}

Aggregation of resources in space can occur at many scales. This article suggests that the impact of aggregation on foraging behavior, and, hence, on lifetime reproduction, may vary profoundly depending on the scale of resource aggregation. Ignoring one scale of aggregation might not tell us the whole story, especially when studying the behavior of individuals. The problem of when and how to incorporate spatial heterogeneity into models is not straightforward. Kareiva (1990) reviewed both theoretical and empirical results from a wide range of spatial problems and concluded that sometimes the spatial distribution matters and sometimes it does not. Keeling (2000) showed how adding spatial structure to models influences the range of parameters under which host-parasitoid populations coexist. To distinguish when spatial distribution does matter, it is critical to understand how different representations of spatial heterogeneity influence predictions of individual behavior and, consequently, the dynamics of populations, communities, and ecosystems. This article contributes to this understanding by simultaneously examining the influence of aggregation at two different scales on the individual behavior and lifetime reproduction of the parasitic wasp, Cotesia rubecula.

As far as we know, this article is the first published work on the influence of resource aggregation at multiple scales
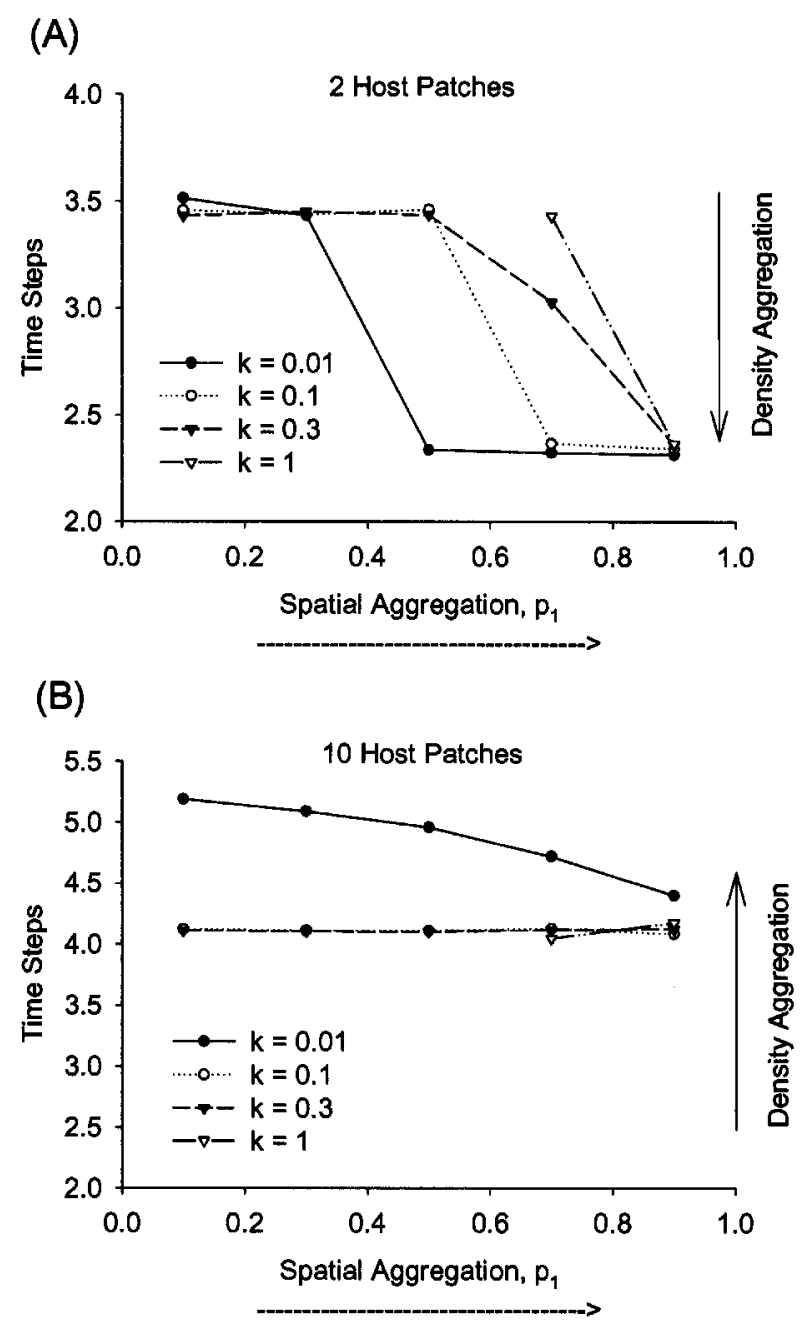

Figure 7: Influence of density aggregation $(k)$ and spatial aggregation $\left(p_{1}\right)$ on mean patch residence time of simulated Cotesia rubecula females on patches with a small $(A)$ and a high $(B)$ number of hosts within a population with a mean density of two hosts per plant. The arrows indicate increasing degree of aggregation. 


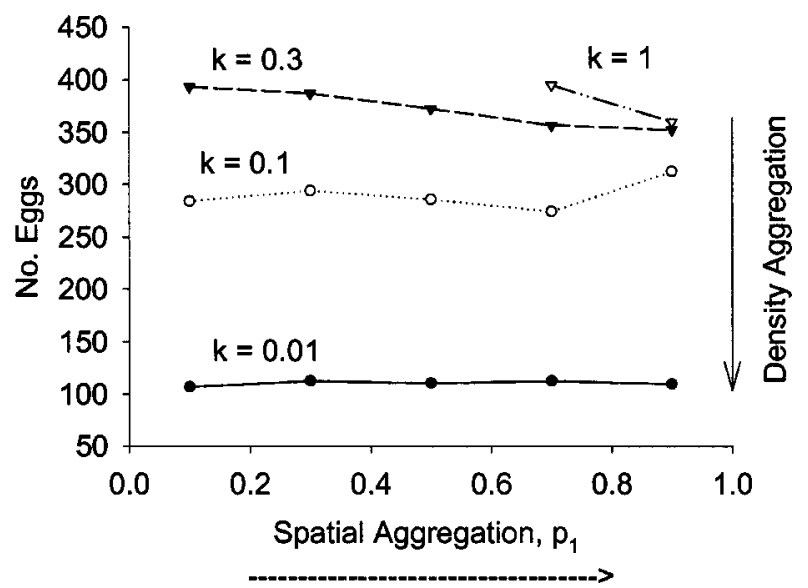

Figure 8: Reproductive success of simulated Cotesia rubecula females as a function of density aggregation $(k)$ and spatial aggregation $\left(p_{1}\right)$. The arrows indicate increasing degree of aggregation.

on the patch-leaving behavior of individuals. Our model explains the influence of aggregation on patch-leaving behavior through its effects on travel time and the relative profitability of host patches. This is entirely consistent with Charnov's (1976) marginal value theorem that predicts predators should stay longer in patches with high profitability and when the travel time is long.

\section{Density Aggregation}

Density aggregation influences the travel time and the relative profitability of patches. With increasing density aggregation, the frequency of both empty and high-density patches increases; the former increases the travel time, and the latter decreases the relative profitability of low-density patches. So increasing density aggregation could have both an increasing and decreasing effect on patch residence time. Our model predicts that wasps respond only at high levels of density aggregation by increasing patch residence time on high-density patches due to increasing travel time through empty patches (fig. 7). At low to moderate levels of density aggregation, the model predicts no influence of density aggregation on patch residence time. Here the effects of changing travel time and relative profitability counteract each other.

\section{Spatial Aggregation}

High spatial aggregation means that there is a high probability of finding a host-occupied patch nearby (short travel time); consequently, we expect a wasp to leave the current patch early. This is exactly what our model wasps do on low-host-density patches and on high-host-density patches at very high degree of density aggregation (fig. 7). However, if wasps are on patches with very high host density and density aggregation is moderate to low, chances are low of encountering a similar good one quickly, and the wasps do not respond to a change in spatial aggregation.

\section{Clarifying Apparent Contradictions to Optimal Foraging Theory}

Most research on foraging behavior concentrates on the effects of density aggregation using functional response models. In these models, the number of prey attacked per unit time by a single predator is a function of prey density (May 1978; Crawley 1992). The proportion of prey that escape from predation is the zero term of the negative binomial distribution: $H_{0}=\left(1+a P_{t} / k\right)^{-k}$, where $a$ is the area of discovery and $P_{t}$ is the parasitoid density at time $t$ (Rogers 1972). The general conclusion of such models is that increasing density aggregation (decreasing $k$ ) decreases resource consumption. Our simulation results confirm this prediction, as the lifetime reproductive output of wasps decreased with increasing degree of density aggregation.

Mols's (1993) criticism of functional response models confused spatial and density aggregation. He simulated the foraging behavior of individual carabid beetles (Pterostichus coerulescens) searching for prey distributed either randomly or aggregated in distinct clusters (20 prey per cluster). In his simulations, the predation rate was higher when prey were confined to clusters compared with when prey were randomly distributed, which is opposite to the predictions of functional response models. He concluded that using the zero term of the negative binomial distribution is not appropriate for estimating predation in aggregated prey distributions. In the light of our results, this contradiction is not surprising because Mols's (1993) work confounds the effects of different scales of aggregation, spatial aggregation in his model, and density aggregation in functional response models.

\section{Conclusions}

In this article, we have investigated the effect of host aggregation at different scales on the optimal foraging behavior and performance of the parasitic wasp C. rubecula. Our model suggests that there is no effect of spatial aggregation on the reproductive success of parasitoids, which lends some support to population models that largely ignore spatial resource aggregation. However, for understanding the foraging behavior of individuals, it is important to distinguish between different scales of 
aggregation because sometimes spatial and density aggregation have opposite effects.

\section{Acknowledgments}

This research was supported by a large Australian Research Council grant (A19532040) to M.A.K. and H.P.P. We thank S. J. Ball, M. Mangel, M. E. Visser, M. Vos, A. T. Wolf, three anonymous reviewers, and the members of the Possingham HyperLab of Ecological Modelling discussion group, in particular I. R. Ball and S. A. Richards, for their comments on earlier versions of the manuscript. We also thank G. Siekmann for providing unpublished data on survival of Cotesia rubecula in field cages.

\section{Literature Cited}

Barker, P. S., and L. B. Smith. 1987. Spatial distribution of insect species in granary residues in the prairie provinces (Canada). Canadian Entomologist 119:1123-1130.

Bellman, R. 1957. Dynamic programming. Princeton University Press, Princeton, N.J.

Bernstein, C., A. Kacelnik, and J. R. Krebs. 1991. Individual decisions and the distribution of predators in a patchy environment. II. The influence of travel costs and structure of the environment. Journal of Animal Ecology 60: 205-226.

Charnov, E. L. 1976. Optimal foraging: the marginal value theorem. Theoretical Population Biology 9:129-136.

Comins, H. N., and M. P. Hassel. 1979. The dynamics of optimally foraging predators and parasites. Journal of Animal Ecology 48:335-351.

Cook, R. M., and S. F. Hubbard. 1977. Adaptive searching strategies in insect parasitoids. Journal of Animal Ecology 46:115-125.

Crawley, M. J. 1992. Population dynamics of natural enemies and their prey. Pages 40-89 in M. J. Crawley, ed. Natural enemies: the population biology of predators, parasites and diseases. Blackwell Scientific, Oxford.

DeBach, P., T. W. Fisher, and J. Landi. 1955. Some effects of meteorological factors on all stages of Aphytis lingnanensis, a parasite of the California red scale. Ecology 36:743-753.

Driessen, G., C. Bernstein, J. J. M. van-Alphen, and A. Kacelnik. 1995. A count-down mechanism for host search in the parasitoid Venturia canescens. Journal of Animal Ecology 64:117-125.

Fink, U., and W. Völkl. 1995. The effect of abiotic factors on foraging and oviposition success of the aphid parasitoid, Aphidius rosae. Oecologia (Berlin) 103:371-378.

Geervliet, J. B. F. 1997. Infochemical use by insect parasitoids in a tritrophic context: comparison of a generalist and a specialist. Ph.D. diss. University of Wageningen.
Godfray, H. C. J. 1987. The evolution of clutch size in parasitic wasps. American Naturalist 129:221-233.

- 1994. Parasitoids: behavioral and evolutionary ecology. Monographs in Behavior and Ecology. Princeton University Press, Princeton, N.J.

Haccou, P., and L. Hemerik. 1985. The influence of larval dispersal in the cinnabar moth (Tyria jacobaeae) on predation by the red wood ant (Formica polyctena): an analysis based on the proportional hazards model. Journal of Animal Ecology 54:755-769.

Haccou, P., S. J. de Vlas, J. J. M. van Alphen, and M. E. Visser. 1991. Information processing by foragers: effects of intra-patch experience on the leaving tendency of Leptopilina heterotoma. Journal of Animal Ecology 60: 93-106.

Harcourt, D. G. 1961. Spatial pattern of the imported cabbageworm, Pieris rapae (L) (Lepidoptera: Pieridae), on cultivated cruciferae. Canadian Entomologist 18: 945-952.

Hassell, M. P., T. R. E. Southwood, and P. M. Reader. 1987. The dynamics of the virburnum whitefly (Aleurotrachelus jelinekii): a case study of population regulation. Journal of Animal Ecology 56:283-300.

Heimpel, G. E., and T. R. Collier. 1996. The evolution of host-feeding behaviour in insect parasitoids. Biological Reviews 71:373-400.

Heimpel, G. E., J. A. Rosenheim, and M. Mangel. 1997. Predation on adult Aphytis parasitoids in the field. Oecologia (Berlin) 110:346-352.

Heimpel, G. E., M. Mangel, and J. A. Rosenheim. 1998. Effects of time limitation and egg limitation on lifetime reproductive success of a parasitoid in the field. American Naturalist 152:273-289.

Hemerik, L., G. Driessen, and P. Haccou. 1993. Effects of intra-patch experience on patch time, search time and searching efficiency of the parasitoid Leptopilina clavipes. Journal of Animal Ecology 62:33-44.

Hubbard, S. F., and R. M. Cook. 1978. Optimal foraging by parasitoid wasps. Journal of Animal Ecology 47: 593-604.

Iwasa, Y., M. Higashi, and N. Yamamura. 1981. Prey distribution as a factor determining the choice of optimal foraging strategy. American Naturalist 117:710-723.

Jervis, M. A., N. A. C. Kidd, and G. E. Heimpel. 1996. Parasitoid adult feeding behaviour and biological control: a review. Biocontrol News and Information 17: 11-26.

Jones, R. E. 1977. Movement pattern and egg distribution in cabbage butterflies. Journal of Animal Ecology 46: 195-212.

- 1987. Behavioral evolution in the cabbage butterfly (Pieris rapae). Oecologia (Berlin) 72:69-76.

Kareiva, P. 1990. Population dynamics in spatially complex 
environments: theory and data. Philosophical Transactions of the Royal Society of London B, Biological Sciences 330:175-190.

Kareiva, P., and N. Shigesada. 1983. Analyzing insect movement as a correlated random walk. Oecologia (Berlin) $56: 234-238$.

Keeling, M. J. 2000. Evolutionary dynamics in spatial hostparasite systems. Pages 271-291 in U. Diekmann, R. Law, and J. A. J. Metz, eds. The geometry of ecological interactions: simplifying spatial complexity. Cambridge University Press, Cambridge.

Kobayashi, S. 1966. Process generating the distribution pattern of eggs of the common cabbage butterfly Pieris rapae crucivora. Researches on Population Ecology 8: 51-61.

Krebs, C. J. 1989. Ecological methodology. Harper \& Row, New York.

Levin, S. A. 1992. The problem of pattern and scale in ecology. Ecology 73:1943-1967.

Lima, S. L., and P. A. Zollner. 1996. Towards a behavioral ecology of ecological landscapes. Trends in Ecology \& Evolution 11:131-135.

Mangel, M., and F. R. Adler. 1994. Construction of multidimensional clustered patterns. Ecology 75:1289-1298.

Mangel, M., and C. W. Clark. 1988. Dynamic modeling in behavioural ecology. Princeton University Press, Princeton, N.J.

May, R. M. 1978. Host-parasitoid systems in patchy environments: a phenomenological model. Journal of Animal Ecology 47:833-843.

McDougall, S. J., and N. J. Mills. 1997. The influence of hosts, temperature and food sources on the longevity of Trichogramma platneri. Entomologia Experimentalis et Applicata 83:195-203.

Mols, P. J. M. 1993. Foraging behaviour of the carabid beetle Pterostichus coerulescens and distributions of prey. II. Wageningen Agricultural University Papers 93-5: 105-201.

Nealis, V. G. 1986. Responses to host kairomones and foraging behavior of the insect parasite Cotesia rubecula (Hymenoptera: Braconidae). Canadian Journal of Zoology 64:2393-2398.

-1990. Factors affecting the rate of attack by Cotesia rubecula (Hymenoptera: Braconidae). Ecological Entomology 15:163-168.

Nealis, V. G., R. E. Jones, and W. G. Wellington. 1984. Temperature and development in host-parasite relationships. Oecologia (Berlin) 61:224-229.

Nelson, J. M., and B. D. Roitberg. 1995. Flexible patch time allocation by the leafminer parasitoid, Opius dimidiatus. Ecological Entomology 20:245-252.

Papaj, D. R., and R. H. Messing. 1996. Functional shifts in the use of parasitized hosts by a tephritid fly: the role of host quality. Behavioral Ecology 7:235-242.

Possingham, H. P., S. Tuljapurkar, J. Roughgarden, and M. Wilks. 1994. Population cycling in space-limited organisms subject to density-dependent predation. American Naturalist 143:563-582.

Rees, N. E., and J. A. Onsager. 1982. Influence of predators on the efficiency of the Blaesoxipha spp. parasites of the migratory grasshopper (Melanoplus sanguinipes). Environmental Entomology 11:426-428.

Roff, D. 1977. Dispersal in dipterans: its cost and consequences. Journal of Animal Ecology 46:443-456.

Rogers, D. 1972. Random search and insect population models. Journal of Animal Ecology 41:369-383.

Roitberg, B. D., and M. Mangel. 1997. Individuals on the landscape: behavior can mitigate landscape differences among habitats. Oikos 80:234-240.

Roitberg, B. D., M. Mangel, R. G. Lalonde, C. A. Roitberg, J. J. M. Van Alphen, and L. Vet. 1992. Seasonal dynamic shifts in patch exploitation by parasitic wasps. Behavioral Ecology 3:156-165.

Rosenheim, J. A., and M. Mangel. 1994. Patch-leaving rules for parasitoids with imperfect host discrimination. Ecological Entomology 19:374-380.

Rosenheim, J. A., H. K. Kaya, L. E. Ehler, J. J. Marois, and B. A. Jaffee. 1995. Intraguild predation among biological-control agents: theory and evidence. Biological Control 5:303-335.

Rosewell, J., B. Shorrocks, and K. Edwards. 1990. Competition on a divided and ephemeral resource: testing the assumptions. I. Aggregation. Journal of Animal Ecology 59:977-1002.

Schneider, D. C. 1994. Quantitative ecology: spatial and temporal scaling. Academic Press, San Diego, Calif.

Stephens, D. W., and J. R. Krebs. 1986. Foraging theory. Princeton University Press, Princeton, N.J.

Stoyan, D., and H. Stoyan. 1994. Fractals, ramdom shapes and point fields. Wiley, Chichester.

Tenhumberg, B., A. J. Tyre, and B. D. Roitberg. 2000. Stochastic variation in food availability influences weight and age at maturity. Journal of Theoretical Biology 202:257-272.

van Alphen, J. J. M. 1993. Patch residence time and encounters with parasitised hosts: a reaction. Netherlands Journal of Zoology 43:340-349.

van Alphen, J. J. M., and L. E. M. Vet. 1986. An evolutionary approach to host finding and selection. Pages 23-61 in J. K. Waage and D. Greathead, eds. Insect parasitoids. Academic Press, London.

van Alphen, J. J. M., M. E. Visser, and H. W. Nell. 1992. Adaptive superparasitism and patch time allocation in solitary parasitoids: searching in groups vs. sequential patch visits. Functional Ecology 6:528-535. 
van Randen, E. J., and B. D. Roitberg. 1996. The effect of egg load on superparasitism by the snowberry fly. Entomologia Experimentalis et Applicata 79:241-245.

Visser, M. E., J. J. M. van Alphen, and H. W. Nell. 1992. Adaptive superparasitism and patch time allocation in solitary parasitoids: the influence of pre-patch experience. Behavioral Ecology and Sociobiology 31:163-171.

Völkl, W. 1992. Aphids and their parasitoids: who actually benefits from ant-attendance? Journal of Animal Ecology 61:273-281.

Völkl, W., and M. Mackauer. 1993. Interactions between ant-attending Aphis fabae spp. cirsiiacanthoidis on thistles and foraging parasitoid waps. Journal of Insect Behavior 6:301-312.
Waage, J. K. 1979. Foraging for patchily-distributed hosts by the parasitoid, Nemeritis canescens. Journal of Animal Ecology 48:353-371.

Wäckers, F. L., and C. P. M. Swaans. 1993. Finding floral nectar and honeydew in Cotesia rubecula: random or directed? Proceedings Experimental and Applied Entomology 4:67-72.

Wiens, J. A. 1989. Spatial scaling in ecology. Functional Ecology 3:385-397.

Wiskerke, J. S. C., and L. E. M. Vet. 1994. Foraging for solitarily and gregariously feeding caterpillars: a comparison of two related parasitoid species (Hymenoptera: Braconidae). Journal of Insect Behavior 7:585-603.

Associate Editor: Per Lundberg 